\title{
Lymph Node Involvement and Related Risk Factors in Patients With Breast Cancer Referred for Radiotherapy: A 20-Year Study on 15000 Women
}

\author{
Fariborz Rousta $^{1 \oplus}$, Maryam Dadashzadeh², Farshad Mahdavi ${ }^{3}$, Ali Reza Nasseri ${ }^{* *}$ \\ Abstract \\ Objectives: Tumor size and axillary lymph node (LN) involvement are used as prognostic markers and a guide for choosing adjuvant \\ therapy. This study aimed to evaluate LN involvement and related risk factors in patients with breast cancer (BC) referred for \\ radiotherapy. \\ Materials and Methods: Using the census sampling method, 15,000 women with BC referring for radiotherapy were enrolled in this \\ retrospective descriptive study performed at Tabriz University of Medical Sciences during 2000-2020. LN involvement and related risk \\ factors were recorded and analyzed using the $t$ test and ANOVA test at a significance level of $P<0.05$. \\ Results: The prevalence of LN involvement in this study was $25 \%$. The estrogen receptor status and LN involvement were associated \\ with metastasis. The odds ratio of metastasis in patients with a negative estrogen receptor was about twice that of women with \\ estrogen receptor positivity. Finally, the risk of metastasis in patients presenting with LN involvement was eight times higher than that \\ of patients without LN involvement. \\ Conclusion: In general, estrogen receptor status and LN involvement were associated with metastasis in patients with BC. Thus, these \\ factors can be regarded as a guide to start necessary interventions earlier in at-risk patients. \\ Keywords: Breast cancer, Lymph nodes, Metastasis, Estrogen receptor
}

\section{Introduction}

Breast cancer (BC) is the most prevalent type of cancer in women, presenting with many complications such as port infection (1), chemotherapy- and surgeryassociated nausea and vomiting (2), sexual problems (3), neuropathic pain (4), post-surgery pain (4), LN-induced edema, and the like, representing $\mathrm{BC}$ as the leading cause of death among women. One of the most important problems in patients with $\mathrm{BC}$ is $\mathrm{LN}$ involvement, which is actually one of the main causes of treatment failure (7). LN involvement, which may provide information about the patient's deteriorating condition, can be associated with prognostic factors such as the tumor size or other tumor characteristics. Nevertheless, the prevalence of LN involvement is still unknown and has been variable in different studies (8).

Axillary LNs are the most common site of involvement in patients with $\mathrm{BC}$ that have spread beyond primary lesions (9). More than half of $\mathrm{BC}$ cases are associated with axillary LN involvement, which plays an important role in the prognosis of $\mathrm{BC}$. Complete $\mathrm{LN}$ resection has been the standard surgical approach for $\mathrm{BC}$ since the twentieth century. Nonetheless, this method is associated with complications such as lymphedema, paresthesia, and vascular and neurological damage $(10,11)$. The tumor size and axillary LN involvement are used to estimate the prognosis of $\mathrm{BC}$ and as guides for selecting appropriate adjuvant therapy. Therefore, this study, which was conducted during a 20-year period, aimed to evaluate LN involvement and related risk factors in patients with $\mathrm{BC}$ referred for radiotherapy.

\section{Materials and Methods \\ Study Design}

This descriptive, retrospective, and cross-sectional study was conducted by assessing the clinical records of all the patients referred to the Radiotherapy Clinic of Shahid Madani hospital (Tabriz University of Medical Sciences) under the supervision of the same physician from the beginning of 2000 to the end of 2020 . The patients were enrolled by the census sampling method. The minimum sample size $(\mathrm{n}=169)$ was calculated based on the results of a similar 10-year study by Ghasemi et al (12) and considering an alpha level of 0.05 , the power of $80 \%$, and a hazard ratio of $16 \%$ for LN involvement. However, all eligible patients $(n=15000)$ fulfilling our inclusion and exclusion criteria were enrolled due to the use of the census sampling method and an increase in the validity 
Key Messages

- LN involvement following BC can increase mortality.

- Awareness of the risk factors of mortality to radiotherapy for $\mathrm{BC}$ can reduce mortality.

- Estrogen receptor status and LN involvement are effective factors in the development of metastasis after breast cancer.

of the data. All the patients were evaluated by the same radiotherapist (a member of the main research team) to ensure data homogeneity.

\section{Inclusion and Exclusion Criteria}

The women with $\mathrm{BC}$, referring to the radiotherapy clinic, with or without axillary LN involvement in the pathological examination, were included in this study. All the patients underwent breast surgery (modified radical mastectomy or breast preservation) and had complete clinical records. The minimum duration of follow-ups was five years for each patient. On the other hand, the exclusion criteria were having no report on axillary LN involvement or not being in pathological examination and having incomplete records.

\section{Study Protocol}

All the patients diagnosed with BC and undergoing LN biopsy, referred to the radiotherapist by a thoracic surgeon (not a member of the research team) from 2000 to 2020, were evaluated based on the study aim. The required data were collected from patients' record, including their age at diagnosis, family history of $\mathrm{BC}$, tumor size $(<2 \mathrm{~cm}$, $2-5 \mathrm{~cm}$, and $>5 \mathrm{~cm})$, disease stage (1-4), LN involvement (present or absent), menopausal status, estrogen receptor status, and progesterone receptor status. Due to multiple missing and unknown variables, the human epidermal growth factor was not included in this model.

All the recorded information was anonymous and kept confidential. The results were presented as means and percentages. All variables were collected into a researchermade instrument that was filled during the first clinical examination, and additional information was recorded in subsequent visits.

\section{Statistical Analysis}

After approval by two researchers in terms of readability and authenticity, the recorded data in each questionnaire were handed to a statistician (not a member of the research team) who then entered the data into SPSS software, version 22. Data were reported using descriptive statistics (i.e., mean, frequency, and percentage) and analyzed by the $t$ test and ANOVA tests while considering a $P<0.05$ as the statistical significance threshold.

\section{Results}

The mean age of participants and the prevalence of LN involvement in this study were $48.19 \pm 5.29$ years and
25\% (3751 patients), respectively. Most of the participants $(6365,42.43 \%)$ had tumor sizes between 2 and $5 \mathrm{~cm}$ and were at grade III of the disease $(5129,34.19 \%)$. Other baseline and demographic information of the participants is presented in Table 1 .

Out of 3751 patients with LN involvement, 2145 (57.19\%) cases were detected with metastases based on radiological findings. Overall, 57.19\% of the participants had both LN involvement and metastasis to other areas (odds ratio, $\mathrm{OR}=3.98, P=0.001$ ) while the remaining cases only had LN involvement (Table 2). The results showed that the OR of metastasis in patients with LN involvement was about four times higher than that of those without LN involvement (95\% confidence interval: 2.85-12.10).

The results of the fit model are presented in Table 3. In this model, all independent variables were initially entered into the model, and then variables with no significant effects were omitted one by one, leaving two variables at the end. However, this model had a lower quasilikelihood information value compared with previous variables, which included estrogen receptor status and LN involvement. The estrogen receptor status was one of the factors affecting metastasis and LN involvement. The OR of metastasis in patients with estrogen receptor negativity was about twice that of women with estrogen receptor positivity. Considering the negative coefficient of estrogen receptor interaction in this model, the impact of the estrogen receptor on metastasis was lower than its impact on LN involvement. The statistical significance of this coefficient also indicated different effects of the estrogen receptor on LN involvement and metastasis. Accordingly, estrogen receptor status did not significantly affect metastasis. LN involvement was close to borderline in both responses. The OR of metastasis in patients with LN involvement was eight times higher than that of patients without LN involvement.

\section{Discussion}

Medical research generally aims to detect the presence or absence of several types of diseases, the impacts of healthy and unhealthy behaviors, the biological effects of exposure to several risk factors, and the like. These responses are usually interrelated, and failure to recognize these relations leads to ineffective estimates and standard errors greater than true values. Using models, it is possible to determine the relationship of each response with an independent variable. Marginal models (average-society) based on generalized estimating equations (GEE) and conditional models with random effects (e.g., mixed generalized models) are among the approaches that are used to assess the fitness of correlated data. The advantage of the GEE method is that it provides moderate estimates of the current condition of society. Thus, when the types of the correlation between responses are unknown, it can be a robust method. In addition, it is computationally 
Table 1. Participants' Baseline and Demographic Information

\begin{tabular}{|c|c|c|c|c|c|}
\hline \multirow{2}{*}{ Variables } & & \multicolumn{4}{|c|}{ Axillary Lymph Node } \\
\hline & & Positive $(n=3751)$ & Negative $(n=11249)$ & $P$ Value & Total $(N=15000)$ \\
\hline Age (Mean \pm SD) & & $50.69 \pm 6.06$ & $47.29 \pm 5.11$ & 0.089 & $48.19 \pm 5.29$ \\
\hline Tumor size $($ Mean $\pm \mathrm{SD})$ & & $6.14 \pm 1.39$ & $4.21 \pm 1.01$ & 0.053 & $5.24 \pm 1.19$ \\
\hline \multirow{3}{*}{$\begin{array}{l}\text { Tumor size } \\
(\mathrm{N}, \%)\end{array}$} & $<2 \mathrm{~cm}$ & $511(13.62 \%)$ & $5125(45.55 \%)$ & \multirow{3}{*}{0.055} & $5636(37.57 \%)$ \\
\hline & $2-5 \mathrm{~cm}$ & $2599(69.28 \%)$ & $3766((33.47)$ & & $6365(42.43 \%)$ \\
\hline & $>5 \mathrm{~cm}$ & $641(17.10)$ & 2358 (20.98) & & 2999 (19.99\%) \\
\hline \multirow{4}{*}{ Grade } & I & $231(6.16 \%)$ & $515(4.57 \%)$ & \multirow{4}{*}{0.486} & $746(4.97 \%)$ \\
\hline & II & $1023(27.27 \%)$ & $3435(30.53 \%)$ & & $4458(29.72 \%)$ \\
\hline & II & $1451(38.68 \%)$ & $3678(32.69 \%)$ & & $5129(34.19 \%)$ \\
\hline & IIII & $1046(27.88 \%)$ & 3621 (31.19) & & $4667(31.11 \%)$ \\
\hline \multirow{9}{*}{ Stage } & 0 & $112(2.98 \%)$ & $345(3.07 \%)$ & \multirow{9}{*}{0.316} & $457(3.05 \%)$ \\
\hline & $1 \mathrm{~A}$ & $314(3.38 \%)$ & $1003(8.91 \%)$ & & $1317(8.78 \%)$ \\
\hline & $1 \mathrm{~B}$ & $456(12.15 \%)$ & $1311(11.65 \%)$ & & $1767(11.78 \%)$ \\
\hline & $2 \mathrm{~A}$ & $689(18.36 \%)$ & $1598(14.20 \%)$ & & $2287(15.24 \%)$ \\
\hline & $2 \mathrm{~B}$ & $854(22.76 \%)$ & $2601(23.12 \%)$ & & $3455(23.04 \%)$ \\
\hline & $3 \mathrm{~A}$ & $319(8.51 \%)$ & $1041(9.26 \%)$ & & $1360(09.06 \%)$ \\
\hline & $3 \mathrm{~B}$ & $421(11.22 \%)$ & $1389(13.34 \%)$ & & $1810(12.06 \%)$ \\
\hline & $3 C$ & $398(10.61 \%)$ & $1125(10.01 \%)$ & & $1523(10.15 \%)$ \\
\hline & 4 & $188(5.02 \%)$ & $836(7.68 \%)$ & & $1024(6.83 \%)$ \\
\hline \multirow{2}{*}{ Family history } & Yes & $2008(53.53 \%)$ & $6029(53.59 \%)$ & \multirow{2}{*}{0.889} & $8037(53.58 \%)$ \\
\hline & No & $1743(46.47 \%)$ & $5220(46.41 \%)$ & & $6963(46.42 \%)$ \\
\hline \multirow{2}{*}{ Menopause } & Yes & $2891(77.07 \%)$ & $8459(75.19 \%)$ & \multirow{2}{*}{0.598} & $11350(75.67 \%)$ \\
\hline & No & $860(22.93 \%)$ & $2790(24.81 \%)$ & & $3650(24.33 \%)$ \\
\hline \multirow{3}{*}{ Site of breast cancer } & Right & $1755(46.78 \%)$ & $5234(46.52 \%)$ & \multirow{3}{*}{0.689} & $6989(46.59 \%)$ \\
\hline & Left & $1681(44.81 \%)$ & $5012(44.55 \%)$ & & $6693(44.62 \%)$ \\
\hline & Both Site & $315(8.41 \%)$ & $1003(8.93 \%)$ & & $1318(8.79 \%)$ \\
\hline \multirow{2}{*}{ Lymphovascular invasion } & Yes & $2145(57.18 \%)$ & $7013(62.34 \%)$ & \multirow{2}{*}{0.473} & $9158(61.05 \%)$ \\
\hline & No & $1606(42.82 \%)$ & $4236(37.65 \%)$ & & $5842(38.95 \%)$ \\
\hline \multirow{3}{*}{ Tumor type } & DCIS & $1622(43.24 \%)$ & $5011(44.54 \%)$ & & $6633(44.22 \%)$ \\
\hline & IDC & $1515(40.38 \%)$ & $4989(44.36 \%)$ & \multirow{2}{*}{0.411} & $6504(43.36 \%)$ \\
\hline & Lobular carcinoma & $314(8.37 \%)$ & $1249(11.10 \%)$ & & $1563(10.42 \%)$ \\
\hline \multicolumn{6}{|l|}{ Pathological factors } \\
\hline \multirow{2}{*}{ Estrogen receptor } & Positive & $2489(66.35 \%)$ & $7003(62.25 \%)$ & \multirow{2}{*}{0.495} & $9492(63.28 \%)$ \\
\hline & Negative & $1262(33.64 \%)$ & $4246(37.75 \%)$ & & $5508(36.72 \%)$ \\
\hline \multirow{2}{*}{ Progesterone receptor } & Positive & $2110(56.25 \%)$ & $6890(61.25 \%)$ & \multirow{2}{*}{0.363} & $9000(60 \%)$ \\
\hline & Negative & $1641(43.47 \%)$ & $4359(38.75 \%)$ & & $6000(40 \%)$ \\
\hline \multirow{2}{*}{ HER2 } & Positive & $1755(46.78 \%)$ & $5378(47.80 \%)$ & \multirow{2}{*}{0.889} & $7133(74.55 \%)$ \\
\hline & Negative & $1996(53.22 \%)$ & $5871(52.20)$ & & $7867(52.45 \%)$ \\
\hline
\end{tabular}

Note. M: Mean; SD: Standard deviation; DCIS: Lobular carcinoma in situ; IDC: Invasive ductal carcinoma; HER2: Human epidermal growth factor receptor 2

Table 2. Metastases in Patients With Breast Cancer and Lymph Node Involvement

\begin{tabular}{lccc}
\hline \multirow{2}{*}{ Lymph Node Involvement } & \multicolumn{3}{c}{ Metastasis } \\
\cline { 2 - 3 } & Yes & No & \multicolumn{2}{c}{$\boldsymbol{P}$ Value } \\
\hline Yes & $2145(57.19 \%)$ & $1606(42.81 \%)$ & 0.001 \\
\hline
\end{tabular}

simpler and applicable in most statistical software. The relative efficiency of the provided estimates by the GEE method has undergone extensive discussion. In this study, a marginal bivariate logistic regression model was applied to investigate local recurrence and metastasis in the obtained data from patients with BC (13-16).

$\mathrm{BC}$ is one of the most common malignancies and it continues to be one of the leading causes of death among women despite advances in early diagnosis and treatment. The overall incidence of cancer seems to rise by increasing age and adopting unhealthy lifestyles (17-20).

Some quantitative studies have assessed the relationship between local recurrence and metastasis. Hosseinzadeh and Haghighat reported that these two events were essentially independent of each other because they occur at different times. Nevertheless, women with local recurrence were at a higher risk of metastasis. They further indicated that the risk of metastasis increased with the incidence of local recurrence although the factors affecting recurrence and metastasis were different. 
Table 3. The Results of the Bivariate Logistic Regression Model Based on a Generalized Estimating Equation With the Responses of LN Involvement and Metastasis

\begin{tabular}{|c|c|c|c|c|c|c|}
\hline \multirow{2}{*}{ Independent Variables } & \multicolumn{3}{|c|}{ Response Variable: LN Involvement } & \multicolumn{3}{|c|}{ Response Variable: Metastasis } \\
\hline & SE & $P$ Value & OR & SE & $P$ Value & OR \\
\hline Axillary LN (Positive) & 0.056 & 2.403 & $(0.55)-0.05$ & 0.095 & 2.382 & (0.44) 0.88 \\
\hline Estrogen receptor (Negative) & $(0.45) 2.25$ & 0.001 & $8 / 305$ & $(0.47) 0.12$ & 0.814 & $1 / 114$ \\
\hline
\end{tabular}

Note. LN: Lymph node; SE: Standard error; OR: Odds ratio; Pos: Positive; Neg; Negative.

Furthermore, the disease-free interval from the beginning of treatment to the occurrence of local recurrence affected the survival rate (8).

In most of the previous studies, estrogen receptor status was negative in participants, and the initial tumor size and LN involvement were introduced as factors affecting recurrence and metastasis (21-25). In all these studies, a separate fit model was developed for each response, which was "time" in most of them. Some studies have also noted that age, human epidermal growth factor, and the duration between surgery and recurrence were among the important factors affecting recurrence and metastasis. In the present study, the results of the bivariate logistic fitting model based on the GEE method for LN involvement and metastasis showed that LN involvement and estrogen receptor negativity were among the contributing factors to LN involvement. On the other hand, LN involvement itself was a prominent predictor of metastasis. These findings are consistent with the results of most previous studies. Nevertheless, the impacts of the primary tumor size and age were not significant on metastasis and local recurrence. Additionally, the fitting model suggested that the impacts of estrogen receptor negativity and LN involvement were more pronounced on the incidence of $\mathrm{LN}$ involvement compared to their effects on metastasis. The results of this study are consistent with the findings of most similar studies in this respect. Overall, this model delivers a more accurate estimation of coefficients and their standard errors, increasing the efficiency of the model (26-28).

Suggestions for Further Research

It is recommended that future studies use this model to identify the risk factors of other interrelated diseases.

\section{Study Limitations}

One of the limitations of this study was patients' incomplete records, leading us to ignore some of the important variables affecting local recurrence and metastasis, including initial tumor size, human epidermal growth factor, and the time interval between surgery and local recurrence.

\section{Conclusions}

In this study, the estrogen receptor status and LN involvement were found to affect the incidence of metastasis in patients with BC. By examining these factors, it is possible to identify at-risk individuals and initiate earlier treatments. Given that the two responses were interrelated with each other, the marginal bivariate logistic regression model provided credible results.

\section{Authors' Contribution}

FR: Study design and data analysis; FM and MD: Data collection and data analysis; AN: Article writing and submission and data analysis.

\section{Conflict of Interests}

Authors declare that they have no conflict of interests.

\section{Ethical Issues}

This study was approved by the Ethics Committee of Tabriz University of Medical Sciences (IR.TBZMED.REC.1397.648). After obtaining the ethical approval and hospital authorities' permission, the researchers referred to the relevant department and recorded the information. All information was anonymous, and the researchers were committed to observe honesty and fidelity in reporting the results.

\section{Financial Support}

This study was financially supported and approved by the Ethics Committee of Tabriz University of Medical Sciences.

\section{Acknowledgments}

The authors would like to thank Tabriz University of Medical Sciences for kind support.

\section{References}

1. Aghamohammadi D, Mehdinavaz Aghdam A, Khanbabayi-Gol M. Prevalence of infections associated with port and predisposing factors in women with common cancers under chemotherapy referred to hospitals in Tabriz in 2015. Iran J Obstet Gynecol Infertil. 2019;21(11):7-13. doi:10.22038/ijogi.2019.12321

2. Eghdam-Zamiri R, Khanbabayi-Gol M. Effects of ginger capsule on treatment of nausea and vomiting in patients receiving cisplatin undergoing mastectomy: a randomized clinical trial. Iran J Obstet Gynecol Infertil. 2020;22(11):15-21. doi:10.22038/ ijogi.2020.14949

3. Khanbabayi-Gol M, Mobaraki-Asl N, Ghavami Z, Zharfi M, Mehdinavaz Aghdam A. Sexual violence against mastectomy women improved from breast cancer. Iran J Obstet Gynecol Infertil. 2019;22(5):52-60. doi:10.22038/ijogi.2019.13582

4. Khanbabayi-Gol M, Rezvani F, Ghavami Z, Mobaraki-Asl N. Prevalence of neuropathic pain and factors affecting sleep quality in women with breast cancer after radiotherapy. Iran J Obstet Gynecol Infertil. 2019;22(6):46-53. doi:10.22038/ ijogi.2019.13743

5. Khanbabayi-Gol M, Dadashzadeh M, Mohammadipour Anvari $\mathrm{H}$. Design and implementation of a checklist for prediction of anesthesia-induced nausea and vomiting in candidate patients for mastectomy. Int J Womens Health Reprod Sci. 2020;8(1):90-94. doi:10.15296/ijwhr.2020.13

6. Aghamohamadi D, Khanbabayi-Gol M. Checklist for determining severity of pain and type and dosage of analgesics administered to patients undergoing breast surgeries. Int J Womens Health Reprod Sci. 2020;8(2):227-231. doi:10.15296/ijwhr.2020.36

7. Khanbabayi-Gol M, Aghamohamadi D. Effect of massage therapy with and without elastic bandaging on pain, edema, and shoulder dysfunction after modified radical mastectomy: a clinical trial. Int 
J Womens Health Reprod Sci. 2020;8(1):73-78. doi:10.15296/ ijwhr.2020.10

8. Hosseinzadeh S, Haghighat S. Determining risk factors for local recurrence and metastasis in breast cancer patients using bivariate logistic model. Journal of Health Promotion Management. 2016;5(5):55-62. doi:10.21859/jhpm-06018

9. Belaid A, Kanoun S, Kallel A, et al. [Breast cancer with axillary lymph node involvement]. Cancer Radiother. 2010;14 Suppl 1:S136-146. doi:10.1016/s1278-3218(10)70017-2

10. Wu JL, Tseng HS, Yang LH, et al. Prediction of axillary lymph node metastases in breast cancer patients based on pathologic information of the primary tumor. Med Sci Monit. 2014;20:577581. doi:10.12659/msm.890345

11. Khanbabayi-Gol M, Eidy M, Zamani Esfahlani M. Frequency ratio of carpal tunnel syndrome in women with breast cancer treated with lymphedema in Tabriz medical education centers; 2018-2019. Iran J Obstet Gynecol Infertil. 2020;22(12):62-68. doi:10.22038/ijogi.2020.15554

12. Ghasemi F, Olfatbakhsh A, Rasekhi AA, Haghighat S. Determining the correlated factors of breast cancer recurrence by Poisson BetaWeibull non- mixture cure model. Iran J Breast Dis. 2020;13(2):818. doi:10.30699/ijbd.13.2.8

13. Hajian S, Mehrabi E, Simbar M, Houshyari M, Zayeri F, Hajian P. Designing and psychometric evaluation of adjustment to illness measurement inventory for Iranian women with breast cancer. Iran J Cancer Prev. 2016;9(4):e5461. doi:10.17795/ijcp-5461

14. Nikkhesal N, Nourizadeh R, Dastgiri S, Mehrabi E. The factors affecting women's decision about unplanned pregnancy: A hierarchical modeling strategy. Int J Womens Health Reprod Sci. 2018;6(4):483-490. doi:10.15296/ijwhr.2018.80

15. Saboktakin L, Barzegar M, Ghorbanihaghjo A, Emamalizadeh M. Study on serum Copper and Zinc level of children with epilepsy during long term therapy with anticonvulsants. Life Sci J. 2012;9(4):1250-1254.

16. Saboktakin L, Rafeey M, Kousha A, Moradi SM. Study on prevalence of Helicobacter pylori infection in adolescents with failure to thrive to compare with control group. Life Sci J. 2012;9(4):1425-1431.

17. Noorizadeh R, Ivanbagha R, Ranjbar Koochaksaraei F, Pezeshki MZ, Bakhtari-Aghdam F. Depression and anxiety in sterilised women in Iran. J Fam Plann Reprod Health Care. 2007;33(4):287. doi:10.1783/147118907782101850

18. Baghianimoghaddam MH, Bakhtari-Aghdam F, Asghari-Jafarabadi M, Allahverdipour H, Dabagh-Nikookheslat S, Nourizadeh R. The effect of a pedometer-based program improvement of physical activity in Tabriz University employees. Int J Prev Med. 2016;7:50. doi:10.4103/2008-7802.177897

19. Alizadeh Sabeg P, Mehrabi E, Nourizadeh R, Poursharifi H,
Mousavi S. The effect of counseling on breast cancer awareness in rural Iranian women: a randomized controlled clinical trial. J Cancer Educ. 2019;34(6):1083-1091. doi:10.1007/s13187-0181411-z

20. Alizadeh-Sabeg P, Mehrabi E, Nourizadeh R, Hakimi S, Mousavi S. The effect of motivational interviewing on the change of breast cancer screening behaviors among rural Iranian women. Patient Educ Couns. 2021;104(2):369-374. doi:10.1016/j. pec.2020.08.011

21. Wu X, Baig A, Kasymjanova G, et al. Pattern of local recurrence and distant metastasis in breast cancer by molecular subtype. Cureus. 2016;8(12):e924. doi:10.7759/cureus.924

22. Cohen O, Lam G, Karp N, Choi M. Determining the oncologic safety of autologous fat grafting as a reconstructive modality: an institutional review of breast cancer recurrence rates and surgical outcomes. Plast Reconstr Surg. 2017;140(3):382e-392e. doi:10.1097/prs.0000000000003576

23. Matin S, Shahbazi G, Tabrizian Namin S, Moradpour R, Feizi F, Piri-Dogahe H. Comparison of placenta PCR and maternal serology of aborted women for detection of Toxoplasma gondii in Ardabil, Iran. Korean J Parasitol. 2017;55(6):607-611. doi:10.3347/ kjp.2017.55.6.607

24. Hashemzadeh K, Dehdilani M, Khanbabayi-Gol M. Study of the effects of simple exercise with or without physiotherapy on prevention of deep vein thrombosis among postmenopausal women requiring coronary artery bypass graft surgery. Int J Womens Health Reprod Sci. 2021;9(1):69-74. doi:10.15296/ ijwhr.2021.12

25. Pourebrahim-Alamdari P, Mehrabi E, Nikkhesal N, Nourizadeh R, Esmaeilpour K, Mousavi S. Effectiveness of motivationally tailored interventions on cervical cancer screening: a systematic review and meta-analysis. Int J Womens Health Reprod Sci. 2021;9(2):8690. doi:10.15296/ijwhr.2021.16

26. Chang JS, Lee J, Chun M, et al. Mapping patterns of locoregional recurrence following contemporary treatment with radiation therapy for breast cancer: a multi-institutional validation study of the ESTRO consensus guideline on clinical target volume. Radiother Oncol. 2018;126(1):139-147. doi:10.1016/j. radonc.2017.09.031

27. Mahvi DA, Liu R, Grinstaff MW, Colson YL, Raut CP. Local cancer recurrence: the realities, challenges, and opportunities for new therapies. CA Cancer J Clin. 2018;68(6):488-505. doi:10.3322/ caac. 21498

28. Lu Y, Chen Y, Zhu L, et al. Local recurrence of benign, borderline, and malignant phyllodes tumors of the breast: a systematic review and meta-analysis. Ann Surg Oncol. 2019;26(5):1263-1275. doi:10.1245/s10434-018-07134-5

(C) 2021 The Author(s); This is an open-access article distributed under the terms of the Creative Commons Attribution License (http:// creativecommons.org/licenses/by/4.0), which permits unrestricted use, distribution, and reproduction in any medium, provided the original work is properly cited. 